\title{
An Optimal Nonlinear Guidance Logic for the Trajectory Tracking of Supercavitating Vehicles
}

\author{
Jia Song and $\mathrm{Ke}$ Gao \\ School of Astronautics \\ Beihang University \\ Beijing 100191, China \\ gaoke@buaa.edu.cn
}

\author{
Erfu Yang \\ Design, Manufacture and Engineering Management \\ University of Strathclyde \\ Glasgow G1 1XJ, UK \\ erfu.yang@strath.ac.uk
}

\begin{abstract}
Supercavitating vehicles (SV) are a class of highspeed autonomous underwater vessels. They present a great challenge in designing the guidance law in comparison with the traditional autonomous underwater vehicles. This is due to the fact that their constraints and working environment are much more complex. To tackle the above challenge, an optimal nonlinear midcourse guidance logic is proposed by considering the wake terminal guidance and the remote target attack tasks. The proposed guidance logic is optimized by using an efficient genetic algorithm to obtain its optimal parameters. The results from our simulation case study suggest that the proposed guidance logic can meet both the motion requirements and navigation constraints while effectively cooperating with the wake terminal guidance. Moreover, it has potential in reducing the energy consumption to significantly improve the overall vehicle energy efficiency.
\end{abstract}

Index Terms - Supercavitating Vehicles, Nonlinear Guidance Logic, Trajectory Tracking, Genetic Algorithm, Parameter Optimisation

\section{INTRODUCTION}

Due to the fast sailing speed and strong penetration ability, the underwater supercavitating vehicles with the capability of supercavitating the drag reduction has received more and more attention. The water contact parts on the supercavitating body are only the cavitation and the rudder surface. Therefore, the losing of most buoyancy is caused by the reduction of the wetted area ${ }^{[1]}$. There is a strong nonlinear effect between the body and the bubble. At the same time, the inclusion of the supercavitating on the vehicle seriously restricts its manoeuvrability [2]. In comparison with the conventional navigation system, these significant differences have brought some great difficulties to the stability and manoeuvres of the supercavitating vehicles.

Several researchers have carried out the relevant research on the related technologies of supercavitating vehicles. In [3] the optimal design of conical cavitator for supercavitating vehicles was studied. Sunho simulated the 2D cavity shape of a coned cavitation ${ }^{[4]}$. On the basis of experiments, the fluid dynamic characteristics of the various cone angles were simulated and analyzed by $\mathrm{Li}^{[5]}$. Mokhtarzadeh studied the influence of the three different shapes on the stability ofvehicle ${ }^{[6]}$. Kim investigated the force of supercavitating vehicles in the transition phase ${ }^{[7]}$. The time delay effect of the bubble change was considered by $\mathrm{Li}$, and the related experiments were carried out ${ }^{[8]}$. The finite element method was used to study the interaction between the manoeuvring process and the cavity ${ }^{[9]}$. In [10], a state feedback controller was designed by using the backstepping method, and the stable control of supercavitating vehicles was realized. An adaptive sliding mode controller was designed based on the guaranteed cost control theory ${ }^{[11]}$. In [12], the adaptive tracking control of supercavitating vehicles was achieved. In [13], the nonlinear model of the fully encapsulated supercavitating vehicles was established and dealt with the feedback linearization. In [14], the cavitation effect was considered in the calculation of the sliding force of the vehicle, and the obstacle avoidance tracking was realized by using the predictive control method. In [15], sliding mode control and linear variable parameter control were used to study the control of supercavitating vehicles. The abovementioned studies have mainly focused on the stability and control of supercavitating vehicles. However, there are few works on the optimal guidance of supercavitating vehicles.

The guidance law will directly affect the hit accuracy. The appropriate guidance law is the basic guarantee for the completion of the vehicle's strike missions. Remote vehicle has the following three guiding phrases, i.e.: the launch phrase, the midcourse phrase, and the terminal phrase. The midcourse guidance phrase serves as a link between the launch phrase and the terminal phrase. It largely depends on the midcourse guidance whether the terminal phase will be proceeded successfully. Therefore, it is critical to design a midcourse guidance law with the quick response and high guidance accuracy for the vehicle guidance system.

In particular, the effective guidance design can decrease the voyage loss and reduce the maneuverability requirements for the energy limited supercavitating vehicles. The supercavitating vehicles move fast during the midcourse guidance phase, which raises some certain demands to restrain the conditions of guidance process and guidance method.

In this study, an optimal midcourse guidance logic is proposed for the vehicle at the terminal stage to enter the wake successfully and meet the interlocking condition between the midcourse phrase and the terminal phrase. Toward this end, an efficient genetic algorithm is used to optimize the parameters of the midcourse guidance logic under the restraint conditions.

\section{GUIDANCE CHARACTERISTICS OF THE SUPERCAVITATING} VEHICLES 
As vessel sails, the rotary motion of its propeller causes the cavitation phenomenon in seawater and make the bubbles rotating backwards. The vessel wake is formed by a long strip area with the large quantities of bubbles and turbulence. The intensity of the wakes generated by the vessel is related to the vessel's speed, tonnage, draft and sea conditions. The intensity of the wakes varies in different regions ${ }^{[16]}$. However, for a specific vessel navigation environment, the distribution of the wake intensity with the relative position of the vehicle $\mathrm{s}$ determined. The bubble size distribution in the wake is similar to that in the ocean, but the bubble density is much higher than that in the ocean ${ }^{[16]}$. The bubbles in the wake of the vessel are exponentially decaying along the wake length. Because of the buoyancy and dissolution of bubbles, the density will decrease rapidly ${ }^{[17]}$.

The wake terminal guidance of supercavitating vehicles use the physics characteristics of the vessel wake to realize the vehicle guidance control. The laser terminal wake homing method for supercavitating vehicles is based on the wake information, which can guarantee the supercavitating vehicles' rapid and accurate hits over targets while gaining the strong robustness and interference immunity. In order to have an optimal attack effect, the supercavitating vehicles should enter into the wake with a special incident angle, which should be solved under the restrict conditions of the midcourse guidance procedure. It is the basic condition guaranteeing the vehicle to follow the wake successfully and fulfill the attacking mission. However, when the vessel is at a remote distance, such as more than 10 kilometers, it has a long distance from the vessel wake, which makes it difficult for the supercavitating vehicles to detect the target and wake position accurately. Furthermore, it is difficult for the vehicle to gain the real-time information about the target movement from a carrier after launching. These factors cause it very difficult to solve the midcourse guidance for the supercavitating vehicles.

In this paper, an optimal midcourse guidance law of the supercavitating vehicles is proposed. Its design is based on the analysis of the general characteristics of the vessel wake flow. As shown in the actual observation, the existence time of thewake lasts from 15 minutes to 45 minutes. The existence time of the wake afforded to the wake guidance lasts 2 minutes to 5 minutes. Before launching, the supercavitating vehicles could achieve a part of the wake trajectory information from the carrier. Therefore, if the vehicles are guided into the vessel wake within its existence time, the part of the wake trajectory could be used as initial target parameters for the midcourse guidance and the criterion whether it meets with the terminal guidance condition. In order to make the full use of this part of the wake trajectory, an appropriate method should be employed to get the vehicle quickly reach the initial point of the vessel wake trajectory after launching. If the guidance law is time-consuming, the vessel wake trajectory may disappear due to the effect of the oceanic condition when the supercavitating vehicles arrive at the initial point of the vessel wake trajectory. Hence, the guidance law should follow the wake information until the wake meets the terminal guidance demand, then the midcourse guidance mission completes.

For the design of the guidance law, the following assumptions are made by considering the midcourse guidance characteristics of the supercavitating vehicles and the geometric characteristics of the wake:

1) The supercavitating vehicles and the target are assumed as particles during the midcourse guidance phrase.

2) The supercavitating vehicles sail in the given depth with their movement limited on the horizontal plane.

3) The geometrical model of the vessel wake is shown in Fig. $1^{[18]}$.

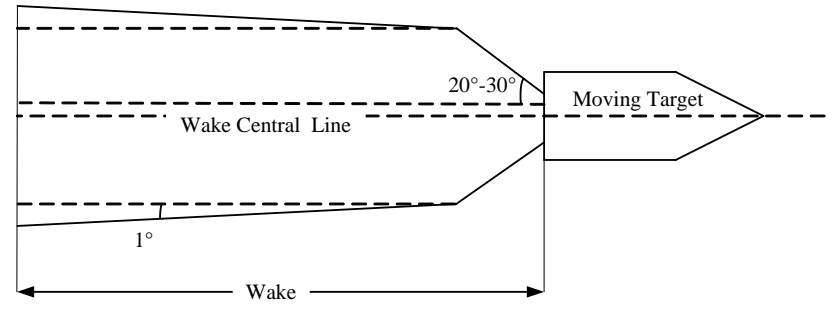

Fig. 1 The geometrical model of vessel wake

As seen in Fig. 1, the divergence angle in the middle of the vessel wake is very small. Therefore, the divergence angle in the middle of the wake is supposed to be zero, which is the end of the wake boundary parallel to the centerline.

4) The centerline direction of the vessel wake coincides with the target track. In other words, the target trajectory obtained through the carrier or other ways can be regarded as the centerline of the wake.

The missile-target coordinate system is established as follows. The initial position of the supercavitating vehicle is defined as the coordinate origin for its midcourse guidance The tangent line of the initial point on the target vessel's wake trajectory is selected as the initial reference line. The Y-axis is in parallel to the reference line and upwards. The $\mathrm{X}$-axis is perpendicular to the $\mathrm{Y}$-axis. The coordinate system is shown in Fig. 2 for the design of midcourse guidance law.

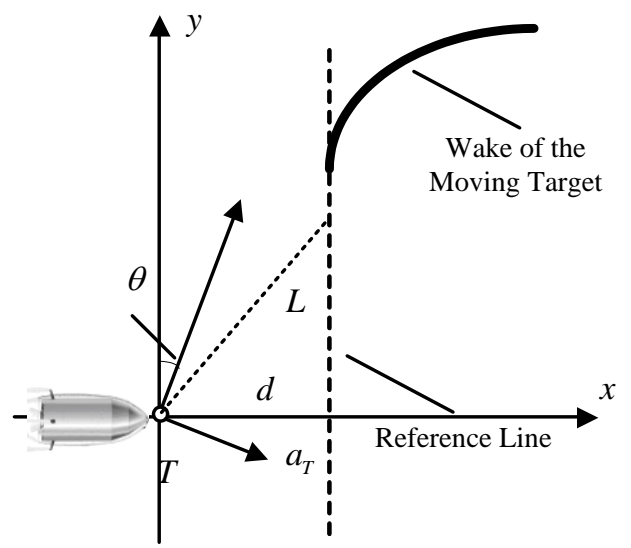

Fig. 2 The Coordinate System for the Design of Midcourse Guidance Law.

As shown in Fig. 2, $V_{T}$ is the speed of the supercavitating vehicle, $\theta$ is the angle between the supercavitating vehicle and the $\mathrm{Y}$-axis, $L$ is the distance between the supercavitating vehicle and a certain point of the target vessel's wake 
trajectory. $d$ is the vertical distance between the supercavitating vehicle and the target trajectory, $a_{T}$ is the acceleration instruction.

The midcourse guidance of the supercavitating vehicle is divided into the following three steps:

1) Firstly, the target's wake trajectory information is obtained before the supercavitating vehicle is launched from its carrier. When the supercavitating vehicle comes into the water, the coordinate relation between the supercavitating vehicle and the target's wake trajectory is built by using the information gained before the launching.

2) Secondly, the tangent line of the initial point on the target's wake trajectory is chosen as the initial reference line, and then is combined with the midcourse guidance law to make the vehicle into the vessel wake as soon as possible.

3) Lastly, when the supercavitating vehicle reaches to the initial point of the vessel wake five points on the target's wake trajectory are chosen by using the bisectional method. The tangent line is made with these points as a new reference line and the designed guidance law is combined to follow the vessel's wake trajectory until the midcourse guidance meets with the terminal guidance. Thus, the midcourse guidance phrase is completed.

\section{GENETIC AlgORITHM BASED OPIMISATION OF THE MIDCOURSE GUIDANCE LAW}

\section{A. Optimal Guidance Law}

According to the characteristics of the supercavitating vehicle's motion, one reference point on the desired trajectory is chosen and the accelerating command is made in terms of the reference point ${ }^{[19]}$. It is supposed that the supercavitating vehicle moves from its original position to the reference point. From the physical characteristics, the centripetal acceleration in the vertical direction can be obtained as $a=V^{2} / R$.

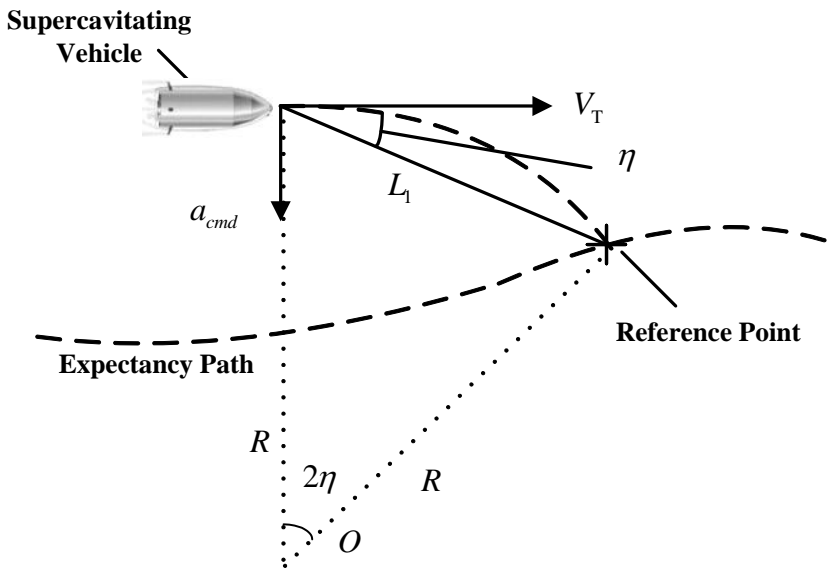

Fig. 3 Schematic Diagram for the Design of Guidance Law

Ffrom Fig. 3, it can be first obtained that that $L_{1}=2 \times R \times \sin \eta$. Then, the lateral acceleration command is determined by

$$
a_{\text {Scmd }}=2 \times\left(V_{\mathrm{T}}^{2} / L_{1}\right) \times \sin \eta
$$

The lateral acceleration command is decided by the distance $L_{I}$ between the vessel and the reference point, and the angle $\eta$ between the vessel's speed direction and $L_{l}$. Combining the coordinate system built in Fig. 2, the midcourse guidance can be expressed as

$$
a_{T}=2 \times V_{T}^{2} \times \sin (\arcsin (d / L)-\theta) / L
$$

As shown in Fig. 2, the direction change of the vehicle's speed $V_{T}$ is decided by the command acceleration and its magnitude remains unchanged. Increasing the command acceleration would increase the speed along the $\mathrm{X}$-axes, which leads the vehicle to approach the target's wake trajectory more quickly. At the same time, it would also decrease the speed in the Y-axes. The time for the vehicle to complete the trajectoryfollowing mission $\mathrm{f}$ is increased.

In order to optimize the magnitude of the command acceleration, the factor $K$ is brought into the proportion and the command acceleration is changed in the following form:

$$
a_{T}=2 K \times V_{T}^{2} \times \sin (\arcsin (d / L)-\theta) / L
$$

It is obvious that the command acceleration is decided by two parameters $K$ and $L$, which should be optimized in the midcourse guidance.

The parameter design problem in the midcourse guidance law can be transformed as an optimization problem that can be solved with a globally-optimal genetic algorithm in the reachability space. The objective function is expressed as follows:

$$
\begin{gathered}
\text { Minimize } f(X)=(\operatorname{det}(J))^{2} \\
\text { Subject to } x_{i} \in S \quad(i=1,2)
\end{gathered}
$$

where $X$ is the impact factor of the command acceleration, $X=\left[\begin{array}{ll}x_{1} & x_{2}\end{array}\right]^{T}=\left[\begin{array}{ll}K & L\end{array}\right]^{T} . S$ is the movement space constraint of the supercavitating vehicle, such as the angular velocity limit and the incidence angle constraint of the midcourse guidance, etc.

\section{B. Optimal Design Based on Genetic Algorithm}

The process of using the genetic algorithm to solve the midcourse guidance problem of the supercavitating vehicles is shown in Fig. 4. 


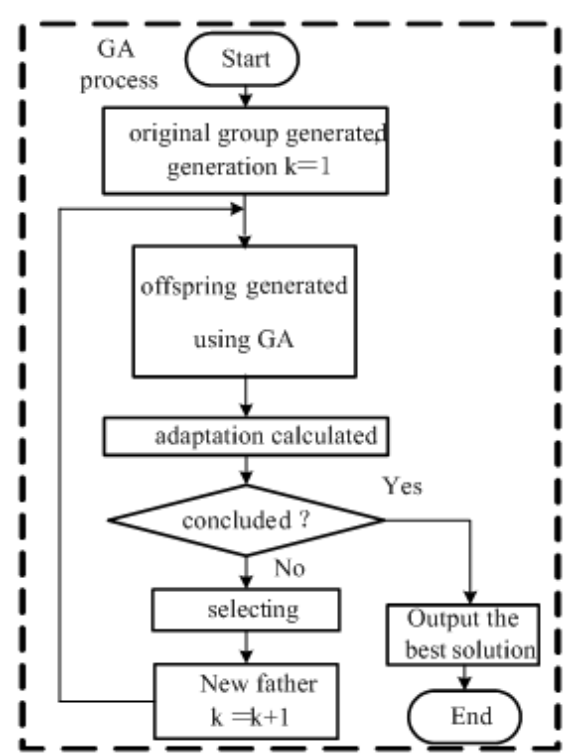

Fig. 4 Genetic algorithm flow chart

1) Coding: Nowadays, the commonly-used coding strategies are the binary coding and the float coding. In this study, if the binary coding is used, the solution space might be too large and the computational efficiency might be reduced. Therefore, a real-valued coding strategy is adopted to construct the chromosome. Each gene represents a floating point of value of the design variable within the chromosome; Each variable is made up by four digits from 0 to 9 . These genes are cascaded together to constitute a chromosome. The float encoding schema is shown in Fig. 5.

The $X$ is expressed as:

\begin{tabular}{|l|c|c|c|c|c|c|c|}
\multicolumn{8}{|c|}{$X=\left[\begin{array}{ll}x_{1} & x_{2}\end{array}\right]^{T}=\left[\begin{array}{ll}K & L\end{array}\right]^{T}$} \\
\hline \multicolumn{8}{|c|}{$K$} \\
\hline$S_{1}$ & $S_{2}$ & $S_{3}$ & $S_{4}$ & $S_{5}$ & $S_{6}$ & $S_{7}$ & $S_{8}$ \\
\hline
\end{tabular}

Fig. 5 Schematic Diagram of the Floating Point Coding

2) Fitness and Selection: Tthe initial population is generated randomly in the solution space, and it is uniformly distributed in the solution space. The population size is taken as $M=100$ in this study. The fitness function denotes the suitability of a solution candidate. In this study, the objective function to be minimized is transformed into the maximization problem of the individual evaluation function:

$$
J(X)=\frac{1}{f(X)+\lambda}
$$

where $\lambda$ is a predefined constant. Its purpose is to avoid the minimum of $E(X)$ being 0 . In this study $\lambda=0.1$.

In this paper, the optimal preservation strategy evolutionary model is used for the survival of the fittest solution. The individuals with the highest fitness in the current population do not participate in the operations of crossover and mutation, but use it to replace the individuals with the lowest fitness after crossover and aberrance.
3) Crossover and Aberrance: Due to the use of the floating point coding strategy, the arithmetic crossover operator is used in this paper to achieve the crossover operation:

$$
X^{\prime}=a X_{1}+(1-a) X_{2}
$$

where $X^{\prime}$ is a descendant of the crossover operation, $X_{1}$ and $X_{2}$ are the parental generation to perform the crossover operations, $a$ is the predefined constant and $a=0.4$. Let the range of the each gene be $\left[L_{i}, U_{i}\right],(i=1,2)$. The uniform mutation operations used in this paper are described as:

$$
\begin{gathered}
m_{j}^{\prime}=\left\{\begin{array}{l}
m_{j}+\Delta\left(t, U_{j}-m_{j}\right), d=0 \\
m_{j}-\Delta\left(t, m_{j}-L_{j}\right), d=1
\end{array}\right. \\
\Delta(k, y)=y r(1-t / G)^{b}
\end{gathered}
$$

where $r$ is a random number between[0,1]. $t$ is the current generation. $G$ is the largest generation number with, $G=250 . b$ is the predefined constant with $b=6.0 . d$ is the probability of the generating random numbers. $m_{j}^{\prime}(j=1,2,3)$ is the filial generation of the $x_{i}^{\prime}$ components gene. $m_{j}(j=1,2,3)$ is the filial generation of the $x_{i}$ components gene.

\section{COMPARATIVE EXPERIMENTAL RESULTS}

In order to evaluate the performance of the proposed guidance law in this work, a series of numerical experiments were conducted. The parameters of the supercavitating vehicles are set as follows. The rotational angular velocity limit is $10^{\circ} / \mathrm{s}^{[20]}$. At the end of the guidance, the distance between the vessel and the centreline of the wake is less than $10 \mathrm{~m}$ and the angle between the velocity direction of the vehicle and the centreline of the wake is less than 10 degrees.

Simulation condition 1: The partial wake trajectory information has been obtained, the initial velocity is $V_{T}=100 \mathrm{~m} / \mathrm{s}$, the initial yaw angle is $\theta=45^{\circ}$, the initial distance of the reference line and the supercavitating vehicle is $d=1000 \mathrm{~m}$. Without using the genetic algorithm to optimize the parameters, $K$ and $L_{1}$ are the constants. The simulation results are shown in Fig.6.

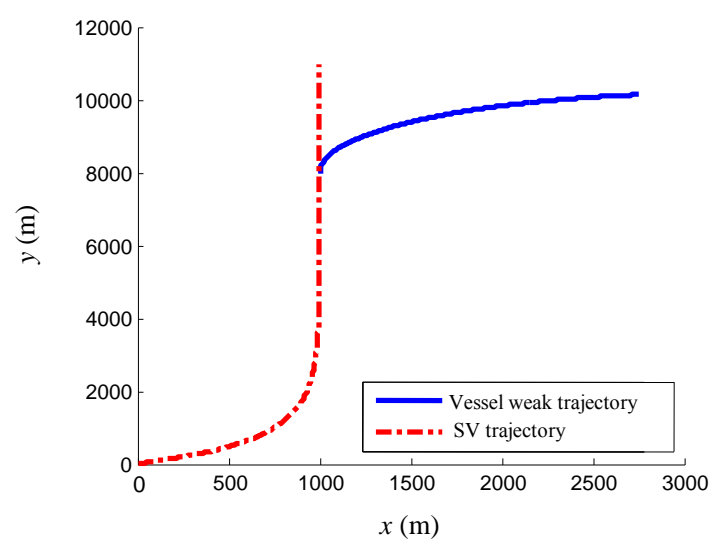

Fig. 6 The guidance trajectories of the Supercavitating vehicle (SV) 


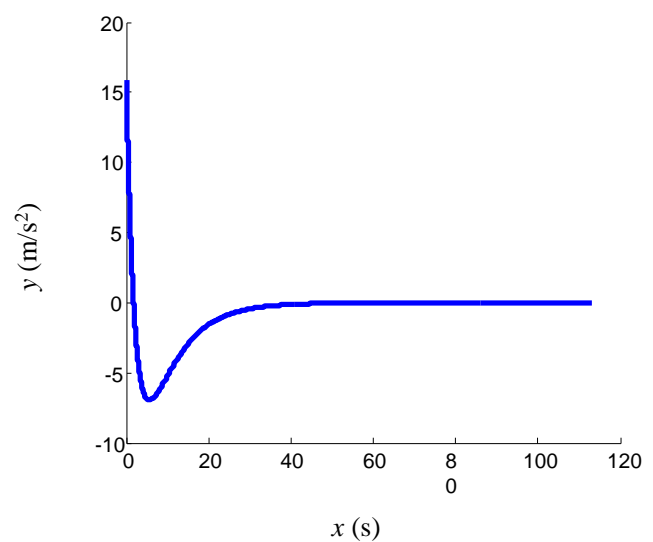

Fig. 7 Acceleration command of the fixed parameter simulation

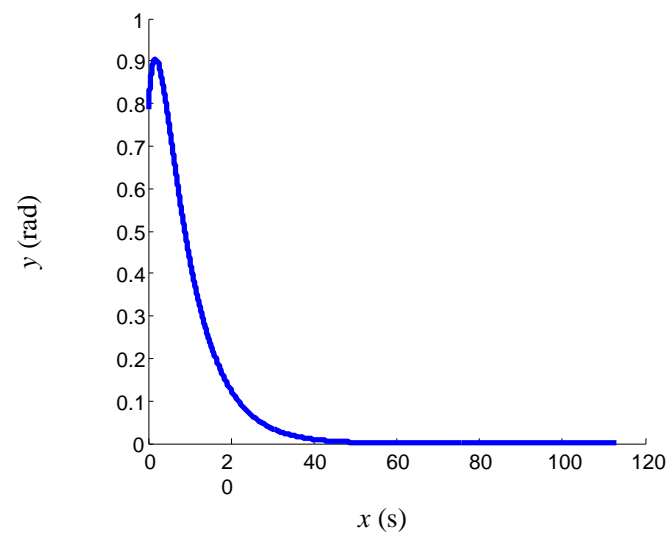

Fig. 8 Yaw angle of the fixed parameter simulation

As shown in Figs.6-.8. when the guidance law's parameters are fixed as constants, the supercavitating vehicle can only reach the initial position of the wake, but the wake-tracking task cannot be completed.

Simulation condition 2: The partial wake trajectory information has been obtained, the initial velocity is $V_{T}=100 \mathrm{~m} / \mathrm{s}$, the initial yaw angle is $\theta=45^{\circ}$, he initial distance of the reference line and the supercavitating vehicle is $d=1000 \mathrm{~m}$. $K$ and $L_{1}$ are optimized by using the genetic algorithm. The simulation results are shown in Figs.9-.13.

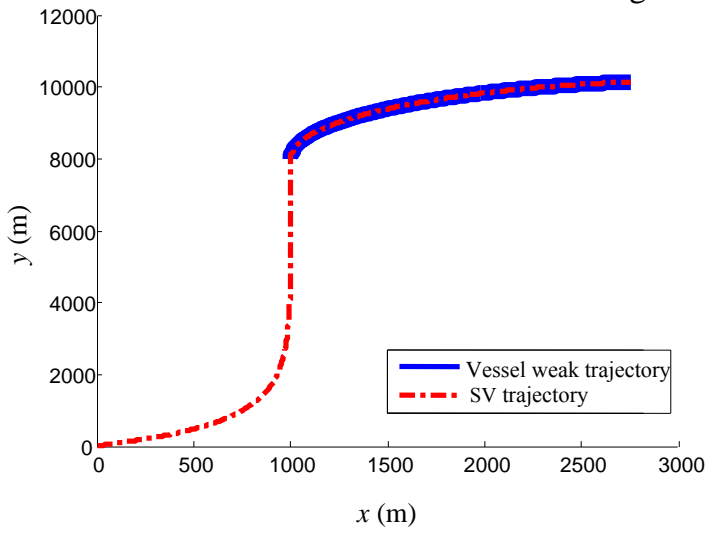

Fig. 9 The guidance trajectories of the supercavitating vehicle (SV)

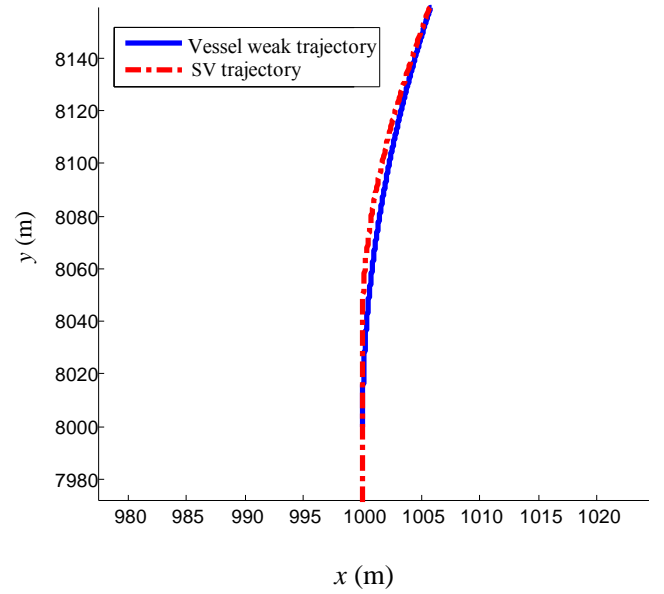

Fig. 10 The trajectories of the SV entering the vessel wake

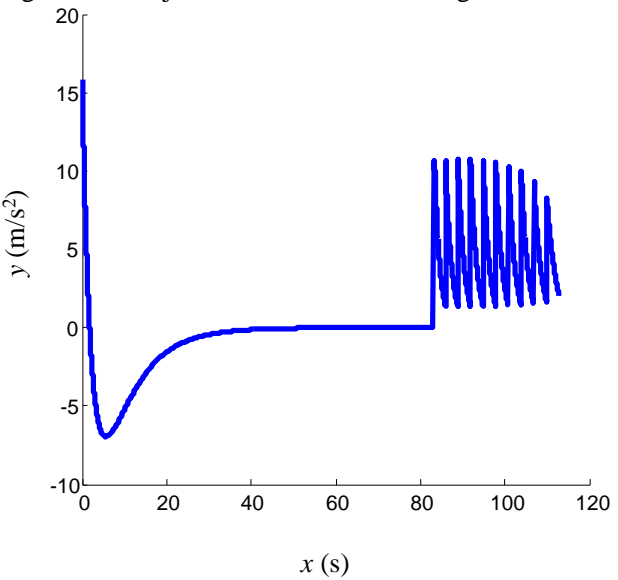

Fig. 11 The ccceleration of the supercavitating vehicle (SV)

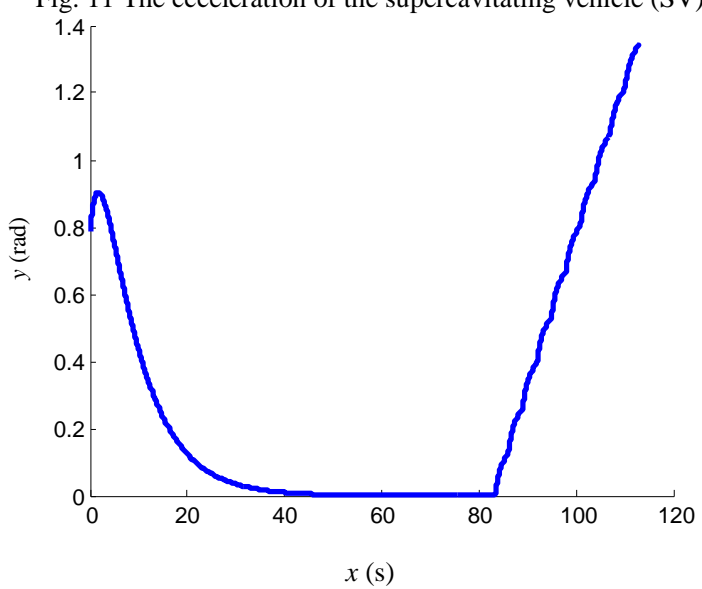

Fig. 12 Yaw angle of the supercavitating vehicle (SV) 


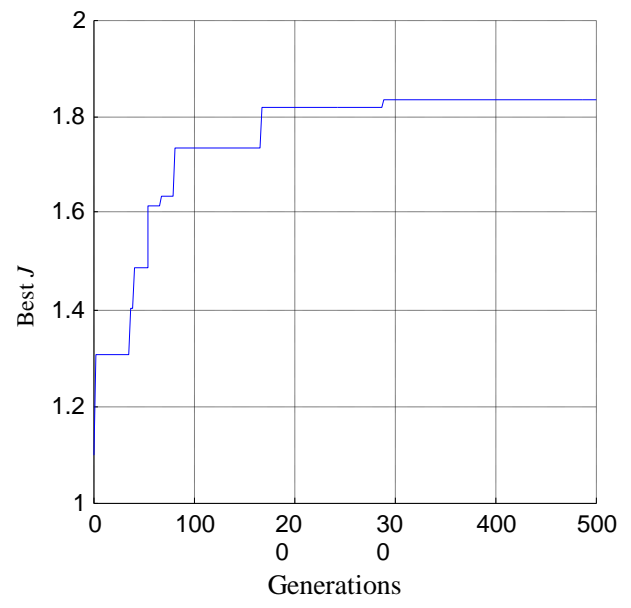

Fig. 13 Genetic Algorithm Optimization Process

As shown in Figs.9- 13, the maximum distance between the wake centerline and the supercavitating vehicle is $5.09 \mathrm{~m}$. The angle between the velocity direction of the supercavitating vehicle and the wake centerline is $4.2^{\circ}$. The simulation lasted for 112.89 seconds. When the supercavitating vehicle tracked the initial reference line, the acceleration instruction of the vehicle changes slightly. During the intermediate segmentation, the acceleration instructions are almost zero since the supercavitating vehicle's velocity direction coincides with the reference line. However, after entering the vessel's wake region, the acceleration instructions change greatly. This is due to the longer initial stages. The trajectory given by the guidance law is relatively flat, and the supercavitating layer is kept with the continuity before the supercavitating vehicle enters the wake. After entering the wake, in order to track the wake and achieve the conditions of homing transfer, the acceleration instructions given by the guidance law change greatly, but it can still be guaranteed within the set of constraints.

Based on the above simulation results, the following conclusions can be drawn:

1) It can be seen from the trajectories of the supercavitating vehicles, under the nonlinear midcourse guidance law, the vehicle is moving rapidly to the tangential direction of the initial point of the wake. The supercavitating vehicle stays in a straight line when it reaches the tangent direction of the wake initial point. When reaching the initial point of the wake, the supercavitating vehicle almost keeps moving along the centerline of the vessel's wake according to the selected tangent reference. Finally, according to the simulation results, the supercavitating vehicle meets the condition of the midcourse and terminal guidance, and the whole guidance process is completed.

2) It can be seen from the simulation time and the distance from the wake centerline, the guidance law optimised by the genetic algorithm can guide the vehicle into the vessel's wake range in the presence of wakes.

3) It can be seen from the simulation results on angle velocity, the turning angle of the vehicle is not saturated during the whole course of guidance. The change rate of turning angle is small before the supercavitating vehicle enters the vessel wake, and the change of turning angle velocity is frequent after the vehicle enters the wake.

\section{CONCLUSION}

In this paper, the midcourse guidance of supercavitating vehicles whose terminal guidance is wake homing was studied for the first time. An optimal nonlinear midcourse guidance law has been designed by using an efficient genetic algorithm. The simulation results have shown that the obtained guidance law meets the constraints of both speed and maneuverability, and the handover of the midcourse guidance and the terminal guidance can be completed successfully.

\section{ACKNOWLEDGMENTS}

This research is funded by the National Natural Science Foundation of China [Grant No. 51206007], the Aeronautical Science Foundation of China [Grant No. 2013ZC51], and the China Scholarship Council (CSC) [Grant No.20140625104]. The valuable suggestions and research supports from colleagues are also greatly appreciated.

\section{REFERENCES}

[1] E.S.David, J.B.Gary, E.A.A.Roger. "Planning avoidance control for supercavitating vehicles," The 2014 American Control Conference. Portland, IEEE, 2014: 4979-4984

[2] I.Kirschner,J.S.Uhlman,J.B.Perkins. "Overview of high speed supercavitating vehicles control”. AIAA Guidance, Navigation, and Control Conference and Exhibit. August 2006.3100-3116

[3] L.Mingdong,H. Fan, Z. Weihua. "Optimal design of conical cavitator of supercavitating vehicles".Journal of National University of Defense Technology,2010,32(4):37-41

[4] P.Sunho, S.Rhee. "Numerical analysis of two-dimensional turbulent super-cavitating flow around a cavitator geometry". 20th AIAA Computational Fluid Dynamics Conference .Hawaii.USA.AIAA.2011.

[5] L.Fuyuan,D.Jianjun,Z.Yuwen. "Influencing factors and characteristics of hydrodynamic of conical cavitator".Journal of Shanghai Jiao Tong University.2016,50(2):246-250.

[6] H.Mokhtarzadeh, G.Balas, R.Arndt. "Effect of cavitator on supercavitating vehicles dynamics". IEEE Journal of Oceanic Engineering, 2012,37( 2) : 156-165.

[7] S.Kim, N.Kim. "Neural network-based adaptive control for supercavitating vehicles in transition phase". Journal of Marine Science and Technology, 2015, 20(3) :454-466.

[8] D. Li,K.Luo,C.Huang C. "Dynamics model and control of high-speed supercavitating vehicles incorporated with time-delay". International Journal of Nonlinear Sciences and Numerical Simulation,2014,15( 3) : 221-230.

[9] K.Yu,G.Zhang,J.Zhou. "Numerical study of the itching motions of supercavitating vehicles". Journal of Hydrodynamics. Ser. B, 2012, 24(6):951-95.

[10] Q.Baochen,H.Yuntao,S.Yao,B.Tao. "Absolute stability control of supercavitating vehicles based on backstepping".IEEE International Conference on Mechatronics and Automation, 2014.1918-1923.

[11] L.Rui, Y.Kaiping, W.Yingiie. "Adaptive robust controller for supercavitating vehicles using guaranteed cost theory". Journal of Harbin Institute of Technology. 2011.18(4):77-81.

[12] X.Mao, Q.Wang. "Adaptive control design for supercavitating vehicles model based on fin force parameter estimation". Journal of Vibration and Control, 2013, 1:2-4.

[13] J.E.Dzielski. "Longitudinal stability of supercavitating vehicles". IEEE Journal of Oceanic Engineering,2011,36( 4):562-570. 
[14] B.Vanek,J.Bokor,G.J.Balas. "Longitudinal motion control of a high speed supercavitation vehicle". Journal of Vibration and Control,2007, 13 (2):159-184.

[15] M.Xiaofeng, W.Qian. "Nonlinear control design for a supercavitating vehicles". IEEE Transactions on Control Systems Technology,2009, 17 (4) :816-832.

[16] M.Balser,C.Harkless ,W.Mclaren. "Bragg-wave scattering and the narrow wake". IEEE Transactions on Geoscience and Remote Sensing, 1998,36 (2) :576-588

[17] Z. Xiangtao, S. Xuwen, Z.Ming. "Simulation of trajectory logic for wake homing torpedo". Torpedo Technology,2009,17(2):40-44.

[18] Z.Jiang,L.Yang. "The present situation of research on shipwake characteristic". Ship Science and Technology,2008,30(4):27-32.

[19] S.Park,J.Deyst J, J.P.How. "A new nonlinear guidance logic for trajectory tracking". AIAA Guidance, Navigation, and Control Conference and Exhibit. Providence, Rhode Island 2004.

[20] C.Min, L.Lehua. "Guidance and control of high-speed supercavitating missile for Germany BARRACUDA". Winged Missiles Journal,2007(5):38-43. 